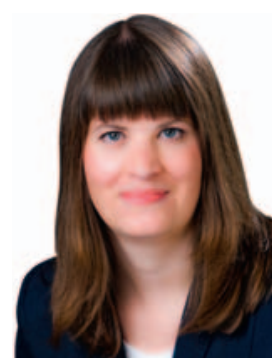

Stephanie Joachim

Koordination «OphthalmoCampus»

\section{Die Zukunft im Blick: Bewährtes nutzen, Visionen entwickeln, Neues schaffen}

Liebe Kolleginnen und Kollegen,

dies ist nun bereits das zweite Mal, dass die Rubrik «OphthalmoCampus» im Karger Kompass OpHTHALMOLOGIE erscheint. Auch die neue Ausgabe beschäftigt sich wieder mit berufspolitischen sowie fachspezifischen Themen, insbesondere für junge Leserinnen und Leser. Schwerpunkt dieser Ausgabe sind die neue Technologien, die Einzug in die Augenheilkunde finden. Es freut mich daher sehr, dass uns Herr Prof. Fischer von der Universitäts-Augenklinik Tübingen einen Einblick in die Möglichkeiten der Gentherapie bei retinalen Erkrankungen gibt.

In Tübingen laufen aktuell die deutschlandweit ersten Gentherapie-Studien an Patienten mit retinalen Erkrankungen. Prof. Fischer spricht darüber, was diese Art der Behandlung in Zukunft für Erkrankungen wie beispielsweise Retinitis pigmentosa oder Makuladystrophien bewirken kann.

Sehr spannend ist auch der Beitrag, in dem Frau Dr. Ullrich uns automatisierte Injektionssysteme in der Augenheilkunde näher bringt. Dr. Ullrich, die kürzlich in die Forbes-Liste der «30 Under 30» (Europe) im Bereich «Science \& Healthcare» aufgenommen wurde, spricht über die Entwicklung und Anwendung von Mikrorobotern für das Auge. Sie forscht an der Möglichkeit, diese für die gezielte Medikamentenabgabe oder für feine mechanische Operationen zu verwenden. Werden wohl intravitreale Injektionen in Zukunft mit einem automatisierten Injektionssystem durchgeführt werden?

Für diese Ausgabe des «OphthalmoCampus» wünsche ich Ihnen viel Spaß beim Lesen!<smiles>CCCCC1CCCCC1C(C)CC</smiles>

\section{KARGER}

Fax +497614520714

information@karger.com www.karger.com (c) 2017 S. Karger GmbH, Freiburg
PD Dr. Stephanie Joachim

Head of Experimental Eye Research

In der Schornau 23-25, 44892 Bochum, Deutschland

stephanie.joachim@rub.de 


\section{S. Karger Verlag für Medizin und Naturwissenschaften $\mathrm{GmbH}$ Literaturrecherche, bibliographische Dienste und Kenngrößen wissenschaftlicher Qualität}

Jeder Wissenschaftler steht bei der Erstellung eigener wissenschaftlicher Studien auf den Schultern von Riesen, will heißen, seine eigene Arbeit baut auf dem auf, was tausende andere vor ihm erforscht und bestimmt haben. Und die Erkenntnisse, die er aus seinen Forschungen zieht, müssen vor dem Kontext bereits vorliegender Befunde bestehen. Doch wie findet man geeignete Literatur für seine Arbeit?

\section{Literaturrecherche}

Wie so vieles ist auch die Literaturrecherche mittlerweile im Wesentlichen internetgestützt. Ein erster Zugang ist die Literatursuche mithilfe klassischer Suchmaschinen wie Google (www.google.de) oder Bing (www.bing.de), die allerdings zu sehr unspezifischen Suchergebnissen führt. Eine deutliche Verbesserung stellt hier schon die Verwendung von Google Scholar (https://scholar.google.de) dar, bei der die Suche schon auf wissenschaftliche Literatur beschränkt ist

Für medizinische Fachliteratur empfiehlt sich allerdings die Verwendung spezieller medizinischer Literaturdatenbanken. Die wahrscheinlich bekannteste und am meisten genutzte dieser Datenbanken ist PubMed (www.ncbi.n/m.nih.gov/pubmed). PubMed wird von der US National Library of Medicine und den National Institutes of Health betrieben. Sie umfasst ausschließlich englischsprachige Literatur. Zudem ist sie fokussiert auf den angelsächsischen Raum, was in Hinblick auf die Betreiber auch nicht verwundern kann. Obwohl sie sehr umfangreich ist, sind bei weitem nicht alle weltweit verfügbaren Journals darin enthalten. Neben ihrer schieren Größe macht diese Datenbank auch ihre Benutzerfreundlichkeit in der Bedienung so beliebt. So lassen sich sehr detaillierte Suchroutinen aufbauen und abspeichern, um sie zu einem späteren Zeitpunkt erneut zu verwenden. Ein auf der PubMed-Seite ver-

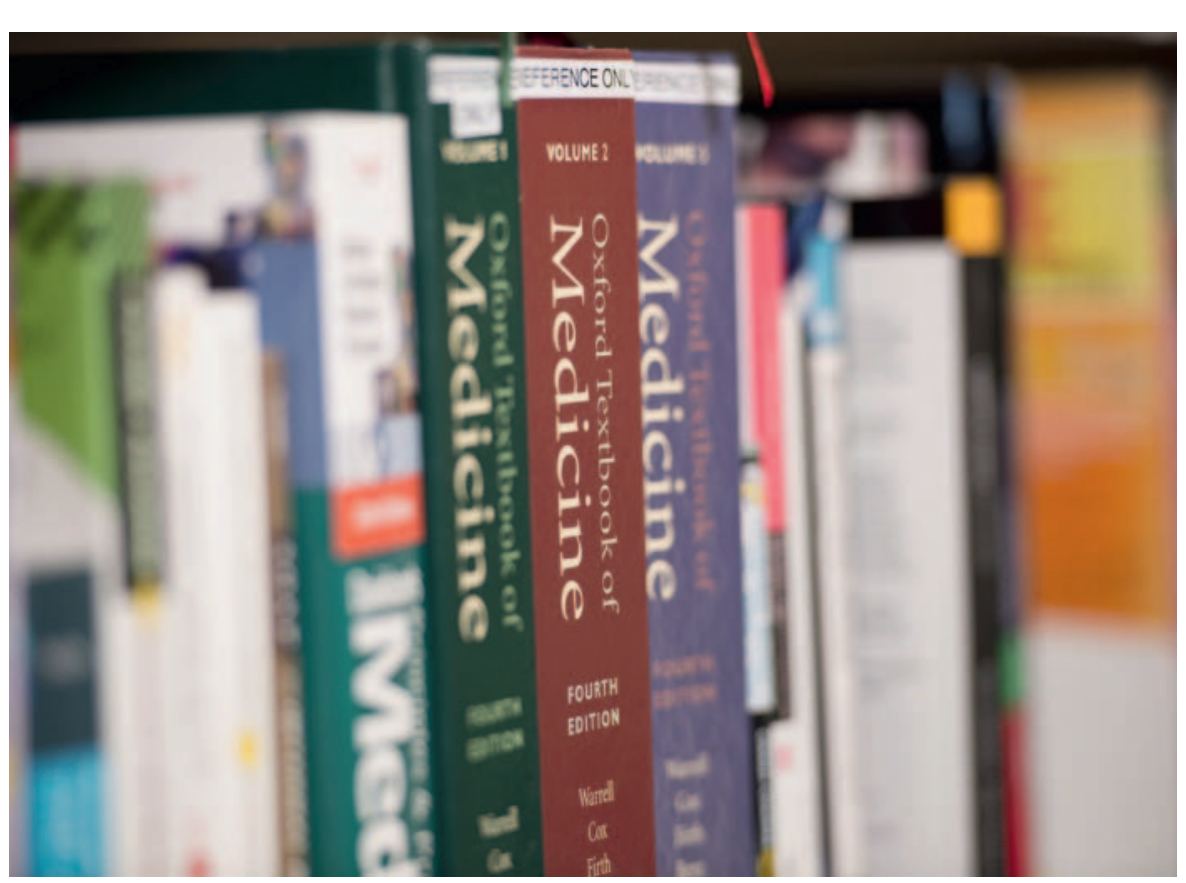

fügbares Video-Tutorial (http://y2u.be/dncRQ1cobdc) führt in einfacher Weise in die Verwendung der erweiterten Suchfunktionen von PubMed ein.

Eine weitere Datenbank, die jeder Mediziner kennen sollte, ist die Cochrane Library. Diese Datenbank umfasst Informationen von hoher Qualität, basierend auf Publikationsformen, die für evidenzbasierte Medizin unverzichtbar sind. Sie wird gepflegt von der Cochrane Collaboration, einem globalem unabhängigem Netzwerk von praktisch tätigen Ärzten, Forschern und Patientenfürsprechern [1]. Obwohl viele der dort verfügbaren Referenzen auch über PubMed auffindbar sind, empfiehlt sich eine separate Suche, da sie, anders als PubMed, kritisch geprüfte Inhalte und darüber hinaus auch Zitationen von primären Datenbanken bietet.

Für eine detaillierte Literatursuche empfiehlt sich somit der Einsatz mehrerer Datenbanken. Je nach Fragestellung ist die eine oder andere besser geeignet, wie der Artikel von Metzendorf et al. [2] eindrucksvoll zeigt.

Eine weitere bedeutende wissenschaftliche Datenbank ist Thomson Reuters Web of Science, die allerdings nicht frei zugänglich ist.

\section{Bewertung wissenschaftlicher Journals und wissenschaftlicher Arbeit}

Als Leser wird man schnell feststellen, dass es große Qualitätsunterschiede zwischen verschiedenen Zeitschriften gibt. Und als Autor wird man sich Gedanken machen, wie gut der eigene Artikel ist, um anhand dessen zu entscheiden, bei welcher Zeitschrift man seinen Artikel einreicht. Je höher das Renommee eines Journals, desto höher das Prestige einer Publikation, aber desto höher auch die Wahrscheinlichkeit, dass ein Artikel nicht zu Publikation angenommen wird und im Begutachtungsverfahren scheitert.

Der wahrscheinlich nach wie vor bedeutendste Indikator für das Renommee einer Zeitschrift ist der vom Thomson Reuters Web of Science ermittelte Impact Factor (IF). Der aktuelle IF berechnet sich wie folgt: Zitate im Jahr 2015 von Publikationen der Jahre $2013+2014$ / Anzahl der Publikationen in $2013+2014$. Für ein noch nicht abgelaufenes Jahr kann es noch keinen IF geben. Der IF 2016 ist erst Mitte des Jahres 2017 verfügbar. Jede Zeitschrift ist einem bestimmten Themenbereich zugeordnet (z.B. «Hematology»), und die Journals werden gemäß ihrem IF in einer Rangliste eingestuft. Natürlich macht es nur Sinn, Zeitschriften eines Themenbereichs zu vergleichen. Der IF ist mittlerweile ein veritabler Wirtschafts- und Einflussfaktor: Bibliotheken orientieren sich bei der Auswahl ihres Zeitschriftensortiments am IF, Forschungsinstitutionen werden anhand des IF ihrer Publikationen bewertet, die Ver- 
gabe von Fördermitteln orientiert sich am IF, Wissenschaftler beurteilen die Qualität ihrer Publikationen anhand des IF und streben die Publikation in Journals mit möglichst hohem IF an.

Eine weitere Messgröße ist der sogenannte Hirsch-Index (h-Index). «Der h-Index ist eine Kennzahl für das weltweite Ansehen eines Wissenschaftlers in Fachkreisen. Die Kennzahl basiert auf bibliometrischen Analysen, d.h. auf Zitationen der Publikationen des Wissenschaftlers. Ein hoher h-Index ergibt sich, wenn eine erhebliche Anzahl von Publikationen des Wissenschaftlers häufig in anderen Veröffentlichungen zitiert ist» [3]. Der h-Index ist stark abhängig von der verwendeten Datengrundlage. Die gängige Datenbasis ist Thomson Reuters Web of Science, die allerdings den Nachteil hat, dass Buchbeiträge nicht erfasst werden, was je nach Forschungsschwerpunkt ein schwerwiegender Nachteil sein kann. Auch andere Datenbanken wie Scopus (www.elsevier.com/solutions/scopus) oder soziale wissenschaftliche Netzwerke wie Research Gate (www. researchgate.net) oder Mendeley (www.mendeley.com) operieren mit einem h-Index.
Dezidierte Kurse zur organisierten Vorbereitung und Durchführung Ihrer wissenschaftlichen Publikation bieten Ihnen Einrichtungen von Universitäten und Arbeitskreise diverser Fachgesellschaften.

Kontaktadresse: Dr. Sven Riestenpatt, Projektmanagement \& Editorial Office Transfusion Medicine and Hemotherapy / Obesity Facts, S. Karger Verlag für Medizin und Naturwissenschaften GmbH, Wilhelmstraße 20a, 79098 Freiburg, Deutschland, s.riestenpatt@karger.com.

\section{Literatur}

1 Timmer A, Richter B: Systematische Übersichtsarbeiten zu Fragen der Therapie und Prävention: Eine Einführung in Frage und Antwort. Teil 4 - Cochrane und die Cochrane Collaboration. Arzneimitteltherapie 2008;26:376-379.

2 Metzendorf M-I, Schulz M, Braun V: All information is not equal: using the literature databases PubMed and the Cochrane Library for identifying the evidence on granulocyte transfusion therapy. Transfus Med Hemother 2014;41:364-374.

3 Wikipedia: h-Index. https://de.wikipedia.org/wiki/H-Index (Zugriff 02.03.2017).

\section{Interview mit Prof. Dr. Dr. M. Dominik Fischer, FEBO \\ Gentherapie: Zukunft der Augenheilkunde}

Die Preisvorlesung «Ophthalmologica Lecture» wird seit 2015 jedes Jahr auf dem Kongress der EURETINA (European Society of Retina Specialists) an junge Wissenschaftler vergeben und im Rahmen des Young Retina Specialists-Symposiums gehalten. Der S. Karger Verlag stellt das Preisgeld in Höhe von 1500 EUR zur Verfügung und veröffentlicht den Vortrag in OPHTHALMOLOGICA, der offiziellen wissenschaftlichen Zeitschrift der EURETINA. Prof. Dr. Dr. M. Dominik Fischer hielt 2015 die erste «Ophthalmologica Lecture» über die Gentherapie in der Ophthalmologie.

\section{Was versteht man unter retinaler Gentherapie?}

Als Gentherapie bezeichnet man das Einfügen von Nukleinsäuren wie RNA oder DNA in die Körperzellen eines Patienten mit dem Ziel, eine Erbkrankheit zu behandeln.

Am besten und sichersten funktioniert das mit einem von der Natur entwickelten Transportersystem. Dieses System, auch Vektor genannt, wird derzeit in unserer Arbeitsgruppe weiter optimiert, um eine bestmögliche Anwendung am Patienten zu erreichen.

Derzeit arbeiten wir mit Vektoren für CNGA3 (Achromatopsie), CHM (Chorioideremie) und PDE6A (Retinitis pigmentosa). Erbkrankheiten entstehen durch ein defektes Gen, also einen Fehler in einem Abschnitt der DNA. Aufgrund dieses Fehlers kann meist ein bestimmter Teil der Körperzelle nicht mehr richtig funktionieren. Abhängig davon, wie bedeutend dieser Funktionsverlust ist, geht die Zelle daran zugrunde.

Als Folge kommt es z.B. bei Erbkrankheiten, welche die lichtwahrnehmenden Zellen (Photorezeptoren) im Auge betreffen, zum fortschreitenden Verlust der Sehfunktion.

Welche besondere Rolle spielt die Ophthalmologie für das Verständnis von Erbmechanismen?

Erbmechanismen wurden zunächst an Pflanzen erkundet (Mendelsche Regeln), treffen aber auch bei Menschen zu. Am Auge werden
Erbkrankheiten oft frühzeitig bemerkt, da das Sehen eine so herausragende Rolle im Alltag spielt. Daher spielte die Genetik in der Augenheilkunde seit jeher eine große Rolle und es wurde früh begonnen, die genetischen Ursachen für erbliche Netzhauterkrankungen zu erforschen.

Über 200 Gene sind inzwischen bekannt dafür, bei Mutationen eine erbliche Netzhauterkrankung auszulösen, die - derzeit unheilbar zu einer Dysfunktion und/oder Degeneration des retinalen Pigmentepithels (RPE), der Stäbchen oder Zapfen und hiermit zu einer Sehbehinderung bis hin zur Erblindung führt. Für eine Handvoll dieser Erkrankungen hat man in der Zwischenzeit gentherapeutische Ansätze bis zum klinischen Einsatz vorangetrieben; eine Leistung, die neben Zeit und Geld eine gute Kooperation zwischen Wissenschaftlern und Ärzten erfordert.

\section{Für welche Krankheiten könnte eine Gentherapie interessant sein?}

Zunächst einmal für alle Erbkrankheiten, bei denen mindestens ein Allel eine Mutation trägt. Am einfachsten erscheint es monogenetische, rezessive Erbkrankheiten wie manche Formen der Retinitis pigmentosa zu behandeln. Aber auch dominante Erbkrankheiten wie z.B. die Mehrzahl der Makuladystrophien oder sogar Netzhauterkrankungen mit mehreren Gendefekten wie die altersbedingte Makuladegeneration könnten unter Umständen von Entwicklungen in der Gentherapie profitieren.

Inwiefern unterscheiden sich gentherapeutische Maßnahmen von anderen Therapieansätzen zur Behandlung von Störungen der Retina? Wie funktioniert die virale Gentherapie? Welche viralen Vektoren eignen sich für die Gentherapie und warum?

Für das Einbringen der therapeutischen Nukleinsäure in die Zielzelle ist ein Vektor, eine «Gen-Fähre», notwendig. Prinzipiell gibt es 
virale und nichtvirale Vektoren. Nichtviraler Gentransfer kann zum einen auf physikalischen Mitteln wie der Elektroporation beruhen, bei der die lipidreiche Zellmembran durch kurze elektrische Impulse für die geladene DNA durchlässiger gemacht wird. Eine andere Möglichkeit sind Nanopartikel, die an das therapeutische Gen gebunden werden. Dieser Nukleinsäurekomplex kann durch rezeptorvermittelte Endozytose in die Zelle aufgenommen werden.

Eine weitere Option sind «Minicircles», welche ebenfalls ohne virale Vektoren auskommen und durch unterschiedliche Methoden der Transfektion wie Elektroporation oder Lipofektion in die Zielzelle gelangen. Minicircles bestehen aus einer zirkulär angeordneten DNA-Sequenz mit komplexer Tertiärstruktur und können eine nahezu beliebig lange therapeutische Sequenz kodieren. Nichtvirale Methoden haben den Vorteil, dass sie vermutlich keine Immunreaktion des Körpers hervorrufen und die Länge der therapeutischen Sequenz nicht beschränkt ist, wie es für manche virale Vektoren der Fall ist. Allerdings sind diese vielversprechenden Methoden des nichtviralen Gentransfers oft nicht sehr wirkungsvoll oder können nur an leicht zugänglichen, unempfindlichen Geweben eingesetzt werden. In der translationalen Forschung haben sich diese Methoden bisher nicht durchgesetzt, auch eine klinische Studie ist dem Autor nicht bekannt.

Beim viralen Gentransfer nutzt man die Tatsache, dass Viren in über 4 Milliarden Jahren evolutionärer Entwicklung die Fähigkeit perfektioniert haben, das ihnen eigene Ge-

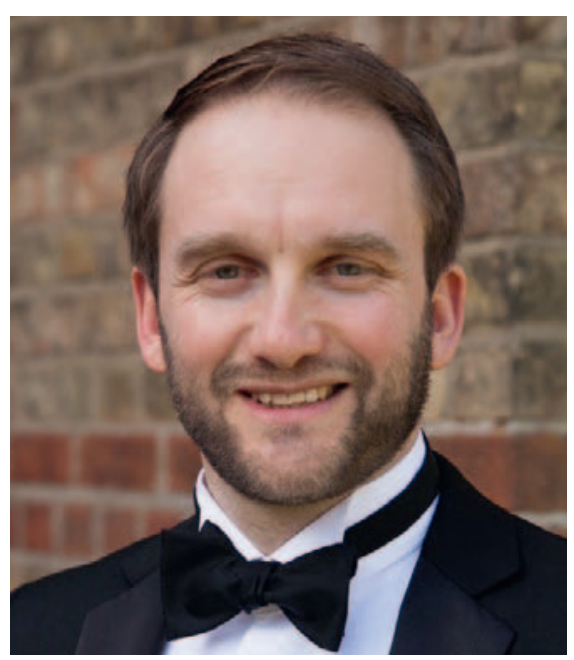

«Die Genetik spielt in der Augenheilkunde seit jeher eine große Rolle»
Welche Herausforderungen ergeben sich aus den multigenetischen Ursachen der Erkrankung für die Diagnostik und Therapie?

Hier besteht die Herausforderung darin, eine therapeutische Nukleinsequenz zu finden, welche die multiplen Ursachen gleichzeitig behandelt. Der Umfang der transportierten Nukleinsäuresequenzen ist bei den meisten Vektoren begrenzt. Auch müssten gegebenenfalls unterschiedliche Dosierungen für unterschiedliche genetische Ursachen berücksichtigt werden.

Wird die retinale Gentherapie bereits angewendet? Welche technischen und ökonomischen Hürden gibt es derzeit? Wo liegen die medizinischen Grenzen der Therapie?

In Tübingen laufen seit November 2015 die deutschlandweit ersten retinalen Gentherapiestudien an Patienten. Diese werden durch die Tistou \& Charlotte Kerstan Stiftung gefördert. Die erste Studie (für Patienten mit Achromatopsie) wurde durch das RD-CURE Konsortium entwickelt und wird weiter in diesem Zusammenhang durchgeführt. Ebenfalls von der Kerstan Stiftung gefördert wird eine weitere Gentherapiestudie für Patienten mit Choroideremie, die auf Vorarbeiten von Professor MacLaren (Oxford, UK) beruht.

Gibt es Patienten, für die sich die Therapie besser eignet als für andere (aufgrund ihres Alters, Geschlechts, Krankheitsstatus etc.)?

Das ist abhängig von der Zielerkrankung und anderen Faktoren. Prinzipiell lässt sich nom in Zielzellen einzuschleusen. Dieser natürliche Mechanismus wird verwendet, nachdem man das virale Genom gegen die therapeutische Sequenz ausgetauscht und das Virus somit zum therapeutischen Vektor umgewandelt hat. Während dem Virus ohne das eigene Genom die Fähigkeit fehlt, sich in der Zielzelle zu replizieren, bleibt die Fähigkeit des Viruspartikels erhalten, über spezifische Liganden auf der Oberfläche des Vektors mit Rezeptoren des Zielgewebes (z.B. fibroblast growth factor receptor 1) zu interagieren, was eine effiziente Clathrin-vermittelte Endozytose ermöglicht. Die vielfältigen viralen Spezies und Serotypen in der Natur weisen durch ihre unterschiedlichen Hüllproteine ein breites Spektrum an Liganden und damit an Gewebespezifität auf. Dieses Spektrum kann durch gezieltes Design der vorkommenden Oberflächenproteine noch erweitert werden. So können rekombinante Serotypen entwickelt werden, die nur ganz spezifische Zellpopulationen transduzieren (z.B. Müller-Zellen, Ganglionzellen, Zapfenphotorezeptoren) oder einen anderen chirurgischen Zugang ermöglichen (intravitreale statt subretinale Applikation). Zellspezifität innerhalb eines Gewebes kann weiter durch regulatorische Einheiten (Promotoren) gewährleistet werden. Ein Beispiel hierfür ist die Zellspezifität des Rhodopsin-Kinase(RK)-Promoters für die Photorezeptoren der Retina. sagen, dass noch Zielgewebe vorhanden sein muss, damit die Gentherapie wirken kann. Komplett erblindete Patienten ohne noch bestehende Restinseln an Netzhautgewebe können am ehesten von einem elektronischen Implantat profitieren, in Zukunft vielleicht aber auch von stammzellbasierten Therapieansätzen.

Ließen sich aus dieser Therapieform auch Behandlungsansätze für andere Erbkrankheiten ableiten?

Unbedingt! Für eine ganze Reihe von anderen Erbkrankheiten wird die Gentherapie bereits entwickelt. Für zwei Krankheiten gibt es sogar schon zugelassene Gentherapeutika: Glybera (Lipoproteinlipase-Defizienz) und Strimvelis (SCID).

Kontaktadresse: Prof. Dr. Dr. M. Dominik Fischer, FEBO, Department für AuElfriede-Aulhorn-Str. 7, 72076 Tübingen, Deutschland, dominik.fischer@ uni-tuebingen.de; Honorary Clinical Research Fellow, Department of Clinical Neurosciences, Nuffield Laboratory of Ophthalmology, University of Oxford, OX3 9DU Oxford, UK. genheilkunde, Universitäts-Augenklinik, Universitätsklinikum Tübingen, 


\section{Junge Mediziner im Blick: Erster Bayerischer Weiterbildungsverbund für die Augenheilkunde}

Ende November 2016 ging der erste augenärztliche Weiterbildungsverbund in Bayern an den Start. Die Bayerische Landesärztekammer erteilte 6 niedergelassenen Augenärzten zusammen mit der Augenabteilung des Klinikums Fürth und dem MVZ Fürth (Ober Scharrer Gruppe) die volle Weiterbildungsberechtigung von 60 Monaten. Diese Idee verfolgte der niedergelassene Augenarzt Dr. Gernot Petzold bereits seit 2013.

Dr. Petzold: «Der Bedarf an gut weitergebildeten Augenärzten in Praxis und Klinik ist stark ansteigend. So bleiben heute freiwerdende Augenarztstellen häufig ohne Bewerber oder müssen mit Ärzten aus dem Ausland nachbesetzt werden. Dies liegt einerseits an der eher abnehmenden Zahl von Weiterbildungsstellen und andererseits an einer nicht unbeträchtlichen Fluktuation der jungen Ärztinnen und Ärzte während ihrer Weiterbildung.»

\section{Augenheilkunde - ein Fachbereich mit viel Dynamik}

In kaum einem anderen medizinischen Fach werden neue diagnostische und therapeutische Verfahren so schnell in die Praxis umgesetzt wie in der Augenheilkunde, so beispielsweise die Entwicklungen in der Diagnostik und Therapie von Netzhauterkrankungen.

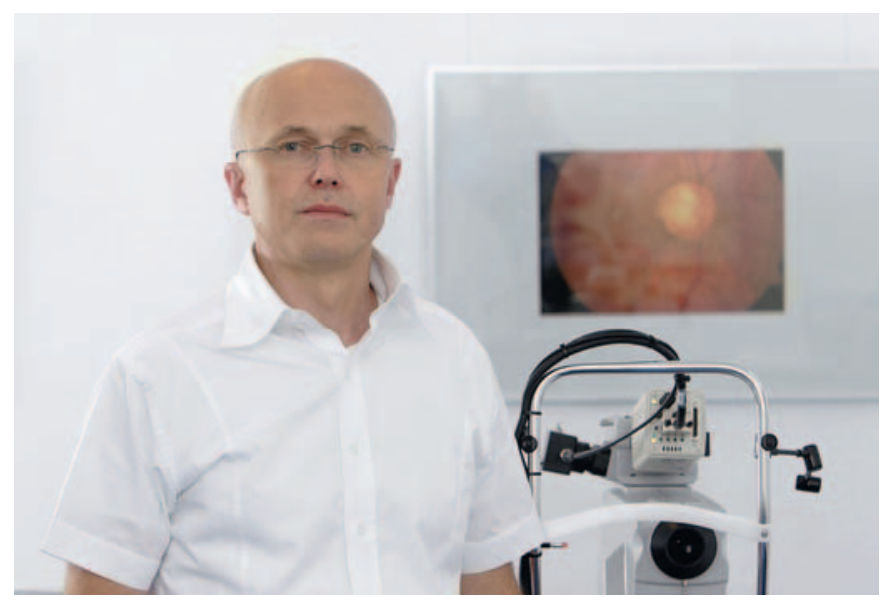

(c) OcuNet.de
Diagnostische und therapeutische Maßnahmen werden seit einigen Jahren überwiegend ambulant durchgeführt. Dies muss auch bei der Weiterbildung berücksichtigt werden. Insofern kann die Vermittlung augenärztlicher Fertigkeiten in vollem Umfang nur durch eine entsprechende Kombination ambulanter und stationärer Weiterbildung erfolgen - idealerweise eng verzahnt.

\section{Umfassende und moderne Weiterbildung}

Junge Mediziner sollen die Möglichkeit erhalten, die Patienten vom Erstkontakt - einschließlich einer zielgerichteten Anamnese, über die Vielzahl der augenärztlichen diagnostischen Möglichkeiten bis hin zur operativen und auch postoperativen Versorgung zu begleiten. Diese strukturierten und aufeinander abgestimmten Weiterbildungsinhalte können nur eng kooperierende ambulante und stationäre Einrichtungen sicherstellen.

Diese Zusammenarbeit von erfahrenen Augenärzten in der Praxis mit herausragenden Ophthalmochirurgen - wie die der Ober Scharrer Gruppe - ist für junge Mediziner besonders attraktiv. Sie erweitern und verbessern hier nicht nur operative Fertigkeiten, sondern lernen auch den besonderen Wert einer vertrauensvollen Arzt-Patienten-Beziehung zu schätzen. Zusätzliche Ausbildungsinhalte sind die Vermittlung von privatarztrechtlichen Prinzipien und von betriebswirtschaftlichen Grundsätzen der Praxisführung sowie der Mitarbeiterführung, des Kassenarztrechts und vielem mehr, was in rein klinischen Weiterbildungseinrichtungen meist unberücksichtigt bleibt.

Dr. Petzold: «Damit hoffen wir, mehr junge Kolleginnen und Kollegen als bisher für den freien Beruf des Augenarztes begeistern zu können.»

Kontaktadresse: Dr. med. Gernot Petzold, Augenarzt, Schatzmeister BFAV/ BVNF, Mitglied der Vertreterversammlung der KVB, Vorsitzender des Kulmbacher Facharztvereins, Hans-Hacker-Straße 1, 95326 Kulmbach, Deutschland, gernot.petzold@t-online.de.

\section{Interview mit Dr. Franziska Ullrich}

\section{Automatisierte Injektionssysteme in der Ophthalmologie - Potenzial für einen Paradigmenwechsel}

Franziska Ullrich wurde 1987 in Göttingen geboren und studierte zunächst Maschineningenieurwissenschaften an der ETH Zürich, mit abschließendem B.Sc. und nachfolgendem M.Sc. in «Robotics, Systems and Controly. Nach mehreren Industriepraktika in der Schweiz, Schweden, China und Australien promovierte sie 2016 am «Multi-Scale Robotics Lab» der ETH Zürich. Sie ist CEO und Mitbegründerin der «Ophthorobotics AG» (www.ophthorobotics.com), die an der Entwicklung medizinischer Roboter arbeitet. Von ihr stammt ein vollautomatisches Roboter-Injektionssystem zur Steigerung von Effizienz und Präzision in der Augenchirurgie. Neben mehreren Auszeichnungen für ihre Arbeit wurde Franziska Ullrich kürzlich in die Forbes-Liste der «30 Under 30» (Europe) im Bereich «Science \& Healthcare» aufgenommen.

\section{Können Sie Ihre Forschung kurz in eigenen Worten erklären?}

Während meines Doktorats erforschte und entwickelte ich verschiedene minimalinvasive Roboter für die Augenchirurgie, sowohl für den vorderen als auch für den hinteren Augenabschnitt. Ein erstes Projekt behandelte die Anwendung von Mikrorobotern für das Auge. Diese winzigen (300 um Durchmesser) zylindrischen Strukturen werden in das Auge injiziert und können dann durch von außen angelegte Magnetfelder genau in 3 Dimensionen gesteuert werden. Die Mikroroboter werden zur gezielten Medikamentenabgabe oder für feine mechanische Operationen im Auge angewendet. In Versuchen an lebenden Hasen konnten wir das hohe Potenzial solcher Mikrostrukturen für die Augenchirurgie demonstrieren. 
Durch eine Kollaboration mit Augenärzten entwickelte sich ein anderes Projekt mit hohem Potenzial für die Augenchirurgie - ein automatisiertes Injektionssystem für die Augen. Dieses System soll Medikamente zur Behandlung der altersbedingten Makuladegeneration und anderer chronischer Netzhauterkrankungen in die Augen injizieren. Es bietet dabei erhöhte Sicherheit für den Patienten und erlaubt es dem Arzt, seine Zeit effizienter zu nutzen. Die Start-up-Firma «Ophthorobotics» hat sich zum Ziel gesetzt, dieses Produkt zur Marktreife zu bringen und anschließend zu kommerzialisieren.

Sie sind in einem äußerst spannenden Lebenslauf von den Ingenieurswissenschaften über die Robotik in die ophthalmologische Medizintechnik gekommen. Gibt es einen roten Faden?

Seit ich 7 Jahre alt war, wollte ich Maschineningenieurin werden. Mein Großvater, ein Maschinenbau- und Verfahrenstechnik-Professor, war immer mein großes Vorbild. In meiner Kindheit habe ich außerdem Spieluhren gesammelt, für mich eine sehr einfache Form eines Roboters. Generell war ich immer schon fasziniert von allem, was sich bewegt. Ich habe also nach dem Schulabschluss Maschinenbau studiert und kam dann auch in Kontakt mit den ersten Robotern. Ich habe mich sofort «verliebt» und bemühte mich um ein Industriepraktikum bei «ABB Corporate Research» in Schweden, wo ich an zweiarmigen Industrierobotern arbeitete. Andere Praktika untermauerten meine Liebe zur Robotik. In Australien konnte ich im «Australian Center for Field Robotics» an einem Mars-Rover arbeiten.

Nach dem Studium habe ich mich zu einer Promotion in der Robotik entschlossen. Meiner Meinung nach ist die Medizinrobotik ein Feld, welches unsere Gesellschaft sehr zum Guten verändern kann! Vielleicht spielte bei dieser Entscheidung indirekt auch meine Familie eine Rolle (Vater und Schwester sind Mediziner, Mutter ist Naturwissenschaftlerin). Ich nahm deshalb eine Doktorandenstelle bei Prof. Nelson am «Multi-Scale Robotics Lab» der ETH Zürich an, wo es um minimalinvasive Systeme für die Augenchirurgie ging. Ich konnte mich gleich für dieses Feld begeistern und hatte Gelegenheit, mehrere Operationen am Auge zu beobachten. Die enge Kollaboration mit Prof. Stephan Michels und Prof. Matthias Becker, stellvertretender Leiter bzw. Leiter der Augenchirurgie des Triemli Spitals in Zürich, erbrachte die Idee zu einem automatisierten Injektionssystem für das Auge.

\section{Wie könnten Ihre Entwicklungen den Praxisalltag von Ophthalmo- logen verändern?}

Das System zur automatisierten Injektion in das Auge soll - im besten Fall - einen Paradigmenwechsel bei Augeninjektionen herbeiführen. Zurzeit stoßen Spitäler an ihre Kapazitätsgrenzen und Patienten laufen Gefahr, nicht mehr ihre optimale Behandlung zu erhalten. Die Anzahl der Patienten, die Injektionen in das Auge benötigen, wächst stetig (4,5 Mio Injektionen in 2014). Dieses Wachstum wird durch die alternde Ge- sellschaft, aber auch durch neuartige Medikamente vorangetrieben. Bei intravitrealen Injektionen, d.h. Injektionen in das Auge, wird häufig die Zeit des Arztes nicht effizient genutzt, da der Arzt während der gesamten Vorbereitung des Patienten anwesend sein muss. Die Injektion selbst ist dann schnell erledigt. Das von uns entwickelte System kann von außerhalb des OP oder Behandlungszimmers gesteuert werden. Das bedeutet, der Arzt bekommt ein Signal, sobald der Patient vorbereitet wurde, und kann dann mit ihm über ein Kommunikationssystem sprechen, alle wichtigen Parameter einstellen und den Injektionsprozess per Knopfdruck starten. Der Patient profitiert von erhöhter Sicherheit durch hochpräzise Injektionen. Außerdem erlaubt eine Iris-Erkennung die eindeutige Identifizierung des Patienten und des Auges, damit keine Verwechslungen passieren können. Dies kommt im stressigen Alltag bei Injektionen leider vor. Der Patient wird natürlich nicht allein gelassen, sondern von Pflegepersonal betreut.

\section{Wenn Sie nochmals entscheiden könnten: Würden Sie den gleichen Weg wählen? Was hätten Sie anders gemacht mit dem Wissen von heute?}

Ich bin mir sicher, ich hätte vieles genauso gemacht. Da auf meinem Lebensweg vieles zum nächsten geführt hat und ich sehr erfüllt von meiner jetzigen Position bin, ist alles richtig gelaufen. Ich würde wieder Maschinenbau und Robotik studieren. Auch von den vielen Auslandsaufenthalten habe ich sehr profitiert. Im Doktorat habe ich viel dazu gelernt, nicht nur fachlich, sondern auch über mich selbst. Natürlich fängt man während seiner Forschung auch Projekte an, die später im Sand verlaufen und trotzdem viel Zeit verschlungen haben. Allerdings bin ich der Meinung, dass auch diese Projekte wichtig sind, um sich selbst auf einen Weg zu bringen und zu positionieren. Ich habe eigentlich nie geplant, nach dem Doktorat eine Firma zu gründen, aber mit der richtigen Idee und den richtigen Partnern schien das ein guter Weg zu sein. Mittlerweile bin ich froh über diese Entwicklung und möchte gar nichts anderes mehr machen. Etwas, das ich durch meine Arbeit gelernt habe, ist, dass man positiv bleiben und Probleme ansprechen muss, bevor sie zu viel Raum einnehmen können.

\section{Was sind Ihre Pläne für die nähere Zukunft?}

Ich möchte das Team und die Firma von «Ophthorobotics» weiter aufbauen und ein klinisch zugelassenes System zur Injektion in die Augen auf den Markt bringen. Dieses System soll nicht nur die Arbeit der Augenärzte erleichtern und dem Patienten ein gutes Erlebnis bei höchster Sicherheit ermöglichen, sondern auch die Kosten für das Gesundheitssystem verringern. Als CEO möchte ich meiner Passion folgen und Robotik entwickeln, die Vorteile für die Gesellschaft bringt.

Kontaktadresse: Dr. Franziska Ullrich, CLAH 7, Tannenstraße 3, 8092 Zürich, Schweiz, fullrich@ethz.ch. 